\title{
Production of a forward $J / \psi$ and a backward jet at the LHC
}

\section{R. Boussarie*}

Institute of Nuclear Physics, Polish Academy of Sciences

Radzikowskiego 152, PL-31-342 Kraków, Poland

E-mail: Renaud.Boussariedifj.edu.pl

\section{B. Ducloué}

Department of Physics, P.O. Box 35, 40014 University of Jyväskylä, Finland

and

Helsinki Institute of Physics, P.O. Box 64, 00014 University of Helsinki, Finland

E-mail: bertrand.b.ducloued jyu.fi

\section{Szymanowski}

National Centre for Nuclear Research (NCBJ), Hoża 69, 00-681 Warsaw, Poland

E-mail: Lech.Szymanowski@ncbj.gov.pl

\section{S. Wallon}

Laboratoire de Physique Théorique (UMR 8627), CNRS, Univ. Paris-Sud, Université

Paris-Saclay, 91405 Orsay Cedex, France

and

UPMC, Université Paris 06, Faculté de Physique, 4 place Jussieu, 75252 Paris, France

E-mail: samuel.walloneth.u-psud.fr

We study the production at the LHC of a forward $J / \psi$ meson and a backward jet with a large rapidity separation using the BFKL formalism. We give predictions for both the Non Relativistic QCD (NRQCD) approach to charmonium production and the Color Evaporation Model. In NRQCD, we find that the ${ }^{3} S_{1}^{8}$ part of the onium wavefunction is completely dominating the process, which makes the presented study a good probe for this color octet contribution.

XXIV International Workshop on Deep-Inelastic Scattering and Related Subjects

11-15 April, 2016

DESY Hamburg, Germany

\footnotetext{
* Speaker.
} 


\section{Introduction}

One of the most promising processes which have been proposed as a way to probe the BFKL [14] resummation effects in the perturbative Regge limit of QCD is the production of two forward jets with a large rapidity interval, as proposed by Mueller and Navelet [12]. Recent $k_{t}$-factorization studies of Mueller-Navelet jets [13-16] were successful in describing such events at the LHC [18]. Here, we propose a similar study for the production of a forward $J / \psi$ meson and a backward jet with a rapidity interval which is large enough to probe the BFKL dynamics but small enough for the meson to be tagged at LHC experiments such as ATLAS or CMS. Although $J / \psi$ mesons were first observed more than 40 years ago, the theoretical mechanism for their production is still to be fully understood and the validity of some models remains a subject of discussions (for recent reviews see for example $[19,20])$. In addition, most predictions for charmonium production rely on collinear factorization. On the contrary, due to the peculiar Mueller-Navelet kinematics, in this work the $J / \psi$ meson and the tagged jet are produced by the interaction of two collinear partons, but with the resummation of any number of accompanying unobserved partons, as usual in the $k_{t^{-}}$ factorization approach.

Here we will compare the two main theoretical descriptions of charmonium production. First we use the NRQCD formalism [21], in which the charmonium wavefunction is expanded as a series in powers of the relative velocity of its constituents. Next we apply the Color Evaporation Model (CEM), which relies on the local-duality hypothesis [22,23]. Finally we will show numerical estimates of the cross section obtained in both approaches. Further details will be provided elsewhere [25].

\section{The scattering cross section in $k_{t}$-factorization}

Within the BFKL formalism for inclusive processes, one writes the cross section as the convolution in transverse momenta of $t$-channel gluons of the impact factor $\Phi_{1}$ for $J / \psi$ meson production, the impact factor $\Phi_{2}$ for the production of the backward jet and the BFKL Green's function $\mathscr{G}$, as illustrated in Fig. 1. Each impact factor is the convolution in the longitudinal momentum fraction space of a parton distribution function (PDF) with the vertex for the fusion of this parton and a $t$-channel BFKL gluon into a $J / \psi$ or a jet. In the NRQCD approache depending on the quantum numbers of the $c \bar{c}$ pair from which the charmonium will be produce, the upper impact factor may or may not take into account the production of a real gluon. In that case since this gluon will not be tagged, its contribution is integrated out.

Thus, introducing the azimuthal angles $\left(\phi_{J / \psi}, \phi_{\mathrm{jet}}\right)$, the rapidities $\left(y_{J / \psi}, y_{\mathrm{jet}}\right)$ and the transverse momenta $\left(\mathbf{k}_{J / \psi}, \mathbf{k}_{\mathrm{jet}}\right)$, we write the differential cross section as follows:

$$
\begin{aligned}
\frac{\mathrm{d} \sigma}{\mathrm{d}\left|\mathbf{k}_{J / \psi}\right| \mathrm{d}\left|\mathbf{k}_{\mathrm{jet}}\right| \mathrm{d} y_{J / \psi} \mathrm{d} y_{\text {jet }}}= & \int \mathrm{d} \phi_{J / \psi} \int \mathrm{d} \phi_{\text {jet }} \int \mathrm{d}^{2} \mathbf{k}_{1} \mathrm{~d}^{2} \mathbf{k}_{2} \mathscr{G}\left(\mathbf{k}_{1}, \mathbf{k}_{2}, \hat{s}\right) \\
& \Phi_{1}\left(\mathbf{k}_{J / \psi}, x_{J / \psi},-\mathbf{k}_{1}\right) \Phi_{2}\left(\mathbf{k}_{\mathrm{jet}}, x_{\mathrm{jet}}, \mathbf{k}_{2}\right) .
\end{aligned}
$$




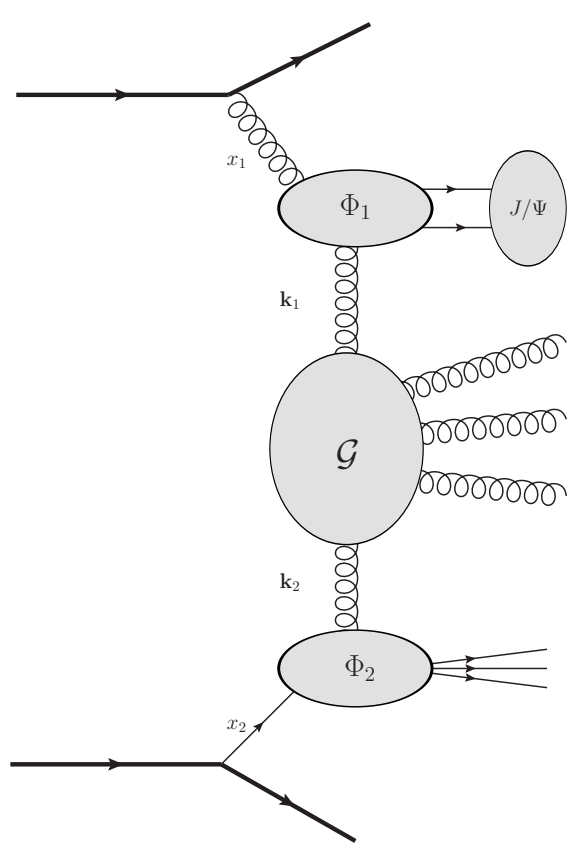

Figure 1: The $k_{t}$-factorized amplitude for the production of a forward $J / \psi$ meson and a backward jet.

\section{Charmonium production in the Non Relativistic QCD formalism}

The NRQCD formalism is based on the static approximation, where the non-perturbative quarkonium wavefunction is expanded in terms of the relative velocity $v \sim \frac{1}{\log M}$ of its constituents. One then postulates that the charmonium production can be factorized into two parts : first, the production of an on-shell $c \bar{c}$ pair is computed using the usual Feynman diagram perturbative methods, then they bind into a charmonium state as encoded in the wavefunction. In the case of an $S$-state charmonium $J / \psi$ with zero orbital momentum one expands the wavefunction as follows :

$$
|\Psi\rangle=O(1)\left|Q \bar{Q}\left[{ }^{3} S_{1}^{(1)}\right]\right\rangle+O(v)\left|Q \bar{Q}\left[{ }^{3} S_{1}^{(8)}\right] g\right\rangle+O\left(v^{2}\right) .
$$

The first term in this expansion corresponds to the production of a quarkonium from a $c \bar{c}$ pair in a color singlet $S^{(1)}$ state. Due to charge parity conservation, the emission of an additional gluon must then be taken into account in the hard part. However, in the second term this additional gluon is included in the wavefunction so it does not appear in the hard part which will then contain only the production of a $c \bar{c}$ pair in a color octet $S^{(8)}$ state. In the inclusive process studied here and to the first order in $v$, both contributions should be included in the cross section.

\subsection{The color singlet contribution}

The hard part which is associated to the first term in Eq. 3.1 consists of six Feynman diagrams, two of which being illustrated in Fig. 2, computed using the color singlet $c \bar{c}$ to $J / \psi$ transition vertex obtained from the NRQCD expansion

$$
v_{\alpha}^{i}\left(q_{2}\right) \bar{u}_{\beta}^{j}\left(q_{1}\right) \rightarrow \frac{\delta^{i j}}{4 N_{c}}\left(\frac{\left\langle\mathscr{O}_{1}\right\rangle_{J / \psi}}{m}\right)^{\frac{1}{2}}\left[\hat{\varepsilon}_{J / \psi}^{*}\left(\hat{k}_{J / \psi}+M\right)\right]_{\alpha, \beta} .
$$


In this equation, $i$ and $j$ are color indices, $\alpha$ and $\beta$ are spinor indices, while $\varepsilon_{J / \psi}$ and $k_{J / \psi}$ are respectively the $J / \psi$ polarization vector and momentum. The $\frac{1}{4 N_{c}}$ factor comes from the projection on spinor indices and on the color singlet. We denote as $m$ the charm quark mass and $M$ the mass of the meson. In the lowest orders in NRQCD the quark and the antiquark carry the same momentum $q$, so that $k_{J / \psi}=2 q$ with $q^{2}=m^{2}$, and one can take $M=2 m$. The operator $\mathscr{O}_{1}$ arises from the non relativistic hamiltonian, and its vacuum expectation value can be fixed by a fit to data, for example from the $J / \psi \rightarrow \mu^{+} \mu^{-}$decay rate.
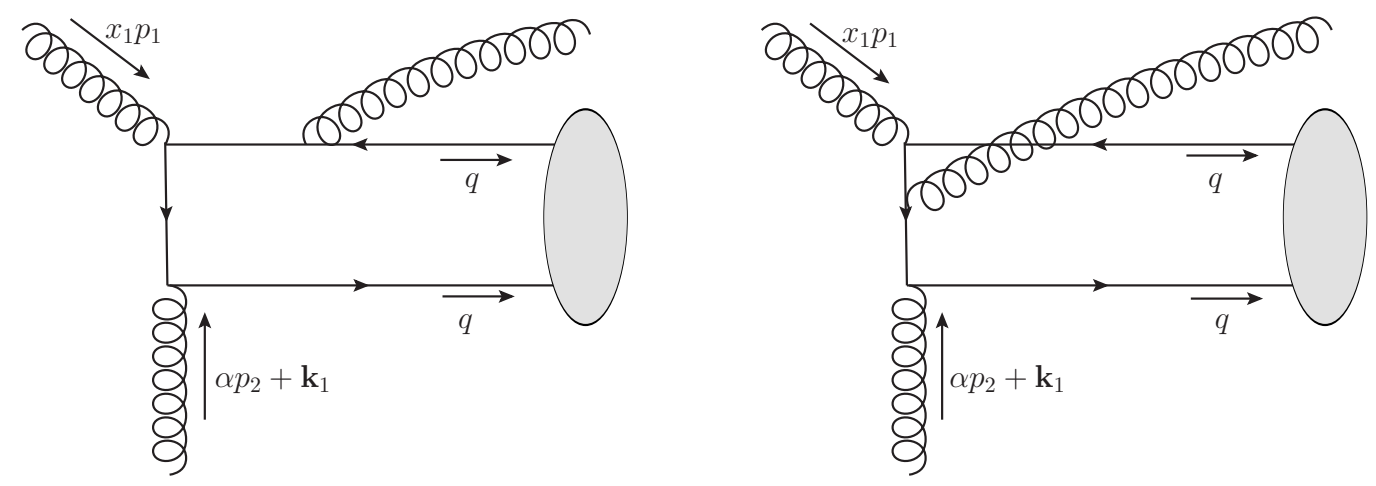

Figure 2: Two examples out of the six diagrams contributing to $J / \psi$ production from a $c \bar{c}$ pair in the color singlet state.

\subsection{The color octet contribution}

The computation of the hard part in the color octet case $[26,27]$ is done in a similar way. It consists of three Feynman diagrams, with two examples shown in Fig. 3. We use the color octet $c \bar{c}$ to $J / \psi$ transition vertex

$$
\left[v_{\alpha}^{i}\left(q_{2}\right) \bar{u}_{\alpha}^{j}\left(q_{1}\right)\right]^{a} \rightarrow \frac{t_{i j}^{a}}{4 N_{c}}\left(\frac{\left\langle\mathscr{O}_{8}\right\rangle_{J / \psi}}{m}\right)^{\frac{1}{2}}\left[\hat{\varepsilon}_{J / \psi}^{*}\left(\hat{k}_{J / \psi}+M\right)\right]_{\alpha, \beta},
$$

where the vacuum expectation value of $\mathscr{O}_{8}$ needs to be determined using experimental data.
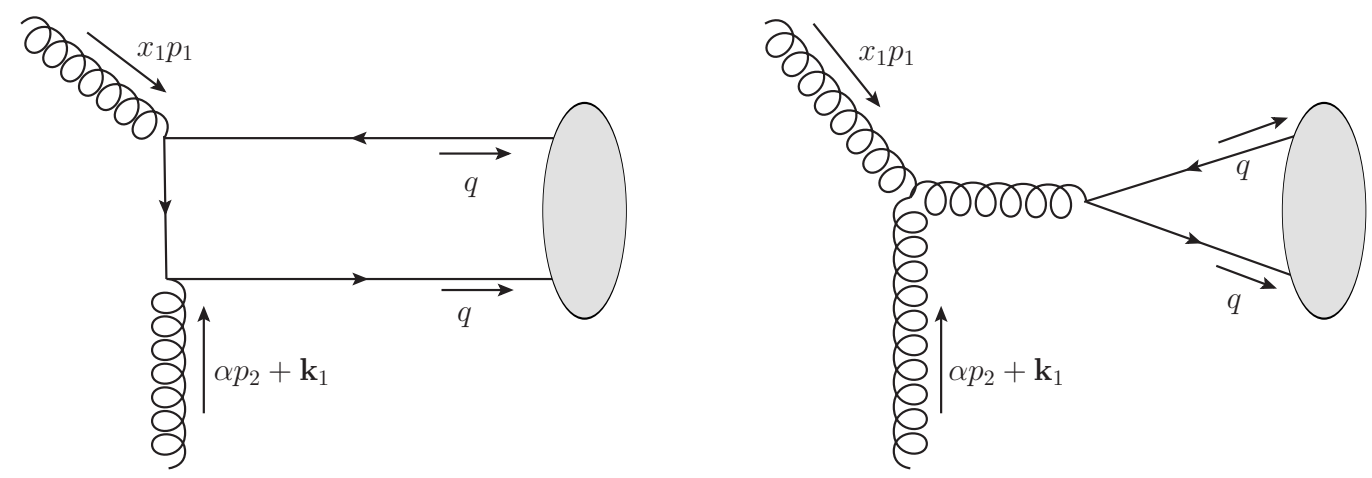

Figure 3: Two examples out of the three diagrams contributing to $J / \psi$ production from a $c \bar{c}$ pair in the color octet state. 


\section{The color evaporation model}

While the NRQCD formalism relies on a factorization hypothesis, the CEM relies on the local duality hypothesis. One assumes that a heavy quark pair $Q \bar{Q}$, with an invariant mass below twice the one of the lightest meson that contains a single heavy quark, will produce a bound $Q \bar{Q}$ state in $\frac{1}{9}$ of the cases, independently of its color. The $\frac{1}{9}=\frac{1}{1+\left(N_{c}^{2}-1\right)}$ factor accounts for the probability for the quark pair to eventually form a colorless state after a series of randomized soft interactions between its production and its confinement. In the case of a charm quark, the upper limit for the invariant mass corresponds to the threshold $2 m_{D}$ for the production of a pair of $D$ mesons. The resulting bound state will correspond to any possible heavy quarkonium. One assumes that the repartition between them is universal.

In other words the cross section for the production of a $J / \psi$ meson will be a fraction $F_{J / \psi}$ of the cross section for the production of a $c \bar{c}$ pair with an invariant mass $M$ between $2 m_{c}$ and $2 m_{D}$, summed over spins and colors

$$
\sigma_{J / \psi}=F_{J / \psi} \int_{4 m_{c}^{2}}^{4 m_{D}^{2}} d M^{2} \frac{d \sigma_{c \bar{c}}}{d M^{2}}
$$

where $F_{J / \psi}$ is assumed to be process-independent and needs to be fitted to data. The required diagrams are similar to the NRQCD color octet diagrams. Let us however emphasize that the quark and the antiquark no longer carry the same momentum, as required to cover the whole range in allowed invariant masses. This is illustrated in Fig. 4.
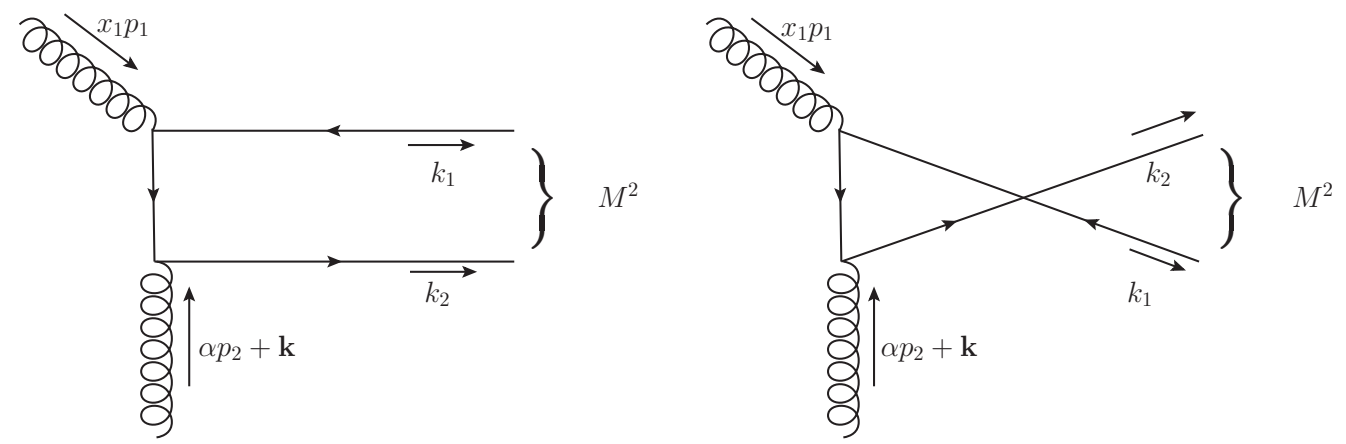

Figure 4: Two examples out of the three diagrams contributing to $J / \psi$ production in the color evaporation model.

\section{Numerical results}

We can now combine the charmonium production impact factor obtained above with the BFKL Green's function and the jet impact factor. Our implementation is very similar to Ref. [15], in particular we use the next-to-leading order jet vertex and the BFKL Green's function at next-to-leading logarithmic accuracy and we use the same scale setting. We note that to perform a complete nextto-leading order study of this process, one would also need to compute the NLO corrections to the charmonium production vertex. In Fig. 5 we show our results for the cross section ${ }^{1}$ as a function

\footnotetext{
${ }^{1}$ Note that these results are different from the previous intermediate results described in Ref. [24]
} 

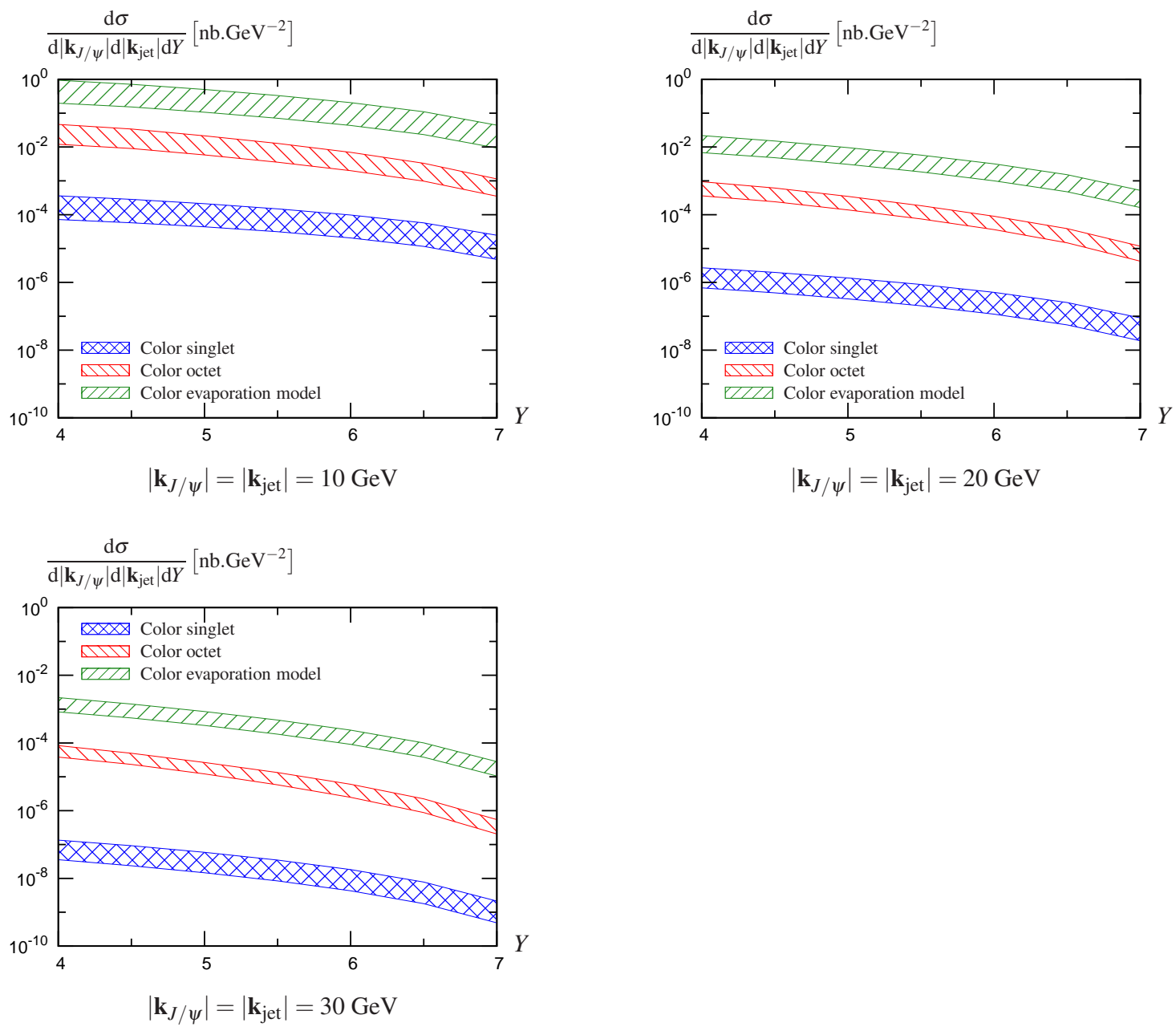

Figure 5: Differential cross section as a function of $Y$ obtained in NRQCD and in the color evaporation model for three values of $p_{T} \equiv\left|\mathbf{k}_{J / \psi}\right|=\left|\mathbf{k}_{\text {jet }}\right|$.

of the rapidity separation between the jet and the $J / \psi, Y \equiv y_{J / \psi}-y_{\text {jet }}$. We use the rapidity cuts $0<y_{J / \psi}<2.5$ and $-4.5<y_{\text {jet }}<0$, which are similar to the acceptances for $J / \psi$ and jet tagging at ATLAS and CMS for example. Here we fix $\left|\mathbf{k}_{J / \psi}\right|=\left|\mathbf{k}_{\text {jet }}\right| \equiv p_{T}$ and we show results for $p_{T}=10,20$ and $30 \mathrm{GeV}$. For the NRQCD calculation we use the same values for $\left\langle\mathscr{O}_{1}\right\rangle$ and $\left\langle\mathscr{O}_{8}\right\rangle$ as in Ref. [28], where they were determined by comparing a $k_{t}$-factorization calculation with experimental data. The value of the CEM parameter $F_{J / \psi}$ extracted from data depends on several details of the calculation, such as the PDF parametrization used. In Ref. [29], values between 0.0144 and 0.0248 are quoted. Here we use a value of 0.02 which is approximately in the center of this interval. We observe from Fig. 5 that in the NRQCD formalism the color singlet contribution is almost negligible compared to the color octet contribution. The cross section in the color evaporation model is of the same order of magnitude as in the NRQCD case, but the two calculations seem to have different behaviours with the kinematics: the decrease of the cross section with increasing $Y$ is slightly more pronounced in the NRQCD approach, while the CEM calculation shows a stronger variation with $p_{T}$. 


\section{Acknowledgments}

We thank E. M. Baldin, A. V. Grabovsky and J.-P. Lansberg for discussions. B. Ducloué acknowledges support from the Academy of Finland, Project No. 273464. This work was partially supported by grant No 2015/17/B/ST2/01838 of the National Science Center in Poland, the PEPSPTI PHENODIFF, the PRC0731 DIFF-QCD, the ANR PARTONS (ANR-12-MONU-0008-01), the COPIN-IN2P3 Agreement and the Theorie-LHC France intiative. This work was done using computing resources from CSC - IT Center for Science in Espoo, Finland.

\section{References}

[1] V. S. Fadin, E. Kuraev, and L. Lipatov, Phys. Lett. B60, 50 (1975).

[2] E. A. Kuraev, L. N. Lipatov, and V. S. Fadin, Sov. Phys. JETP 44, 443 (1976).

[3] E. Kuraev, L. Lipatov, and V. S. Fadin, Sov. Phys. JETP 45, 199 (1977).

[4] I. Balitsky and L. Lipatov, Sov. J. Nucl. Phys. 28, 822 (1978).

[5] H. Cheng and T. T. Wu, Phys. Rev. D1, 3414 (1970).

[6] G. Frolov and L. Lipatov, Sov. J. Nucl. Phys. 13, 333 (1971).

[7] V. Gribov, G. Frolov, and L. Lipatov, Yad. Fiz. 12, 994 (1970).

[8] S. Catani, M. Ciafaloni, and F. Hautmann, Phys. Lett. B242, 97 (1990).

[9] S. Catani, M. Ciafaloni, and F. Hautmann, Nucl. Phys. B366, 135 (1991).

[10] J. C. Collins and R. K. Ellis, Nucl. Phys. B360, 3 (1991).

[11] E. M. Levin, M. G. Ryskin, Yu. M. Shabelski, and A. G. Shuvaev, Sov. J. Nucl. Phys. 53, 657 (1991), [Yad. Fiz.53,1059(1991)].

[12] A. H. Mueller and H. Navelet, Nucl. Phys. B282, 727 (1987).

[13] D. Colferai, F. Schwennsen, L. Szymanowski, and S. Wallon, JHEP 1012, 026 (2010), [hep-ph/1002.1365].

[14] B. Ducloué, L. Szymanowski, and S. Wallon, JHEP 1305, 096 (2013), [hep-ph/1302.7012].

[15] B. Ducloué, L. Szymanowski, and S. Wallon, Phys. Rev. Lett. 112, 082003 (2014), [hep-ph/1309.3229].

[16] B. Ducloué, L. Szymanowski, and S. Wallon, Phys. Lett. B738, 311 (2014), [hep-ph/1407.6593].

[17] B. Ducloué, L. Szymanowski, and S. Wallon, Phys. Rev. D92, no.7, 076002 (2015), [hep-ph/1507.04735].

[18] CMS, V. Khachatryan et al., JHEP 1608, 139 (2016), [hep-ph/1601.06713].

[19] N. Brambilla et al., Eur. Phys. J. C71, 1534 (2011), [hep-ph/1010.5827].

[20] G. T. Bodwin et al., Quarkonium at the Frontiers of High Energy Physics: A Snowmass White Paper, in Community Summer Study 2013: Snowmass on the Mississippi (CSS2013) Minneapolis, MN, USA, July 29-August 6, 2013, 2013, [hep-ph/1307.7425].

[21] G. T. Bodwin, E. Braaten, and G. P. Lepage, Phys. Rev. D51, 1125 (1995), [hep-ph/9407339], [Erratum: Phys. Rev.D55,5853(1997)]. 
[22] H. Fritzsch, Phys. Lett. B67, 217 (1977).

[23] F. Halzen, Phys. Lett. B69, 105 (1977).

[24] R. Boussarie, B. Ducloué, L. Szymanowski, and S. Wallon, Proceeding for the Photon2015 conference, [hep-ph/1511.02181].

[25] R. Boussarie, B. Ducloué, L. Szymanowski, and S. Wallon, in preparation.

[26] P. L. Cho and A. K. Leibovich, Phys. Rev. D53, 150 (1996), [hep-ph/9505329].

[27] P. L. Cho and A. K. Leibovich, Phys. Rev. D53, 6203 (1996), [hep-ph/9511315].

[28] P. Hagler, R. Kirschner, A. Schafer, L. Szymanowski, and O. V. Teryaev, Phys. Rev. D63, 077501 (2001), [hep-ph/0008316].

[29] M. Bedjidian et al., Hard probes in heavy ion collisions at the LHC: Heavy flavor physics, 2004, [hep-ph/0311048]. 